\title{
THE FREE EXACT CATEGORY ON A LEFT EXACT ONE
}

\author{
A. CARboni and R. CElia magno
}

(Received 24 September 1981)

Communicated by R. H. Street

\begin{abstract}
We give an explicit one step description of the free (Barr) exact category on a left exact one. As an application we give a new two step construction of the free abelian category on an additive one.
\end{abstract}

1980 Mathematics subject classification (Amer. Math. Soc.): 18 E 10.

\section{Introduction}

Following a suggestion of A. Joyal, we construct the free exact category $\mathbf{A}_{\mathrm{ex}}$ on a left exact one $\mathbf{A}$ by adding as new objects the equivalence relations in $\mathbf{A}$ and as new arrows between the new objects suitable classes of "compatible maps"; in this way equivalence relations gain quotients in the new category, but the problem is that this process gives rise to new equivalence relations which do not necessarily have coequalizers. We will show that to avoid this obstruction it suffices to consider pseudo equivalence relations (see Section 1 below). The appropriate universal property of our construction is that, for each exact category $\mathbb{E}$, we have an equivalence of categories between the category of left exact functors from $A$ to $\mathbf{E}$ and the category of exact functors from $\mathbf{A}_{\mathrm{ex}}$ to $\mathbb{E}$. This amounts to showing that the construction of $\mathbf{A}_{\mathrm{ex}}$ defines a left biadjoint to the inclusion of the 2-category $\delta x$ of exact categories and exact functors into the 2-category $\mathscr{E} e x$ of left exact categories and left exact functors, which, moreover, make the doctrine $\mathbf{M}$ it induces on Lex into a "Kock-Zöberlein doctrine" in the terminology of [4], such that the $\mathbf{M}$-algebras are precisely the exact categories. As an application, by using

C Copyright Australian Mathematical Society 1982 
Tierney's theorem [2] that an additive exact category is abelian, we use the construction $\boldsymbol{A}_{\mathrm{ex}}$ to produce a new two step description of the free abelian category on an additive one, perhaps a bit less mysterious than that contained in [1].

\section{Pseudo equivalence relations}

An exact category in the sense of Barr [2] is a left exact one (that is, with finite limits) such that:

a) every morphism $f: A \rightarrow B$ has a regular epi-mono factorization;

b) regular epis are pullback stable;

c) every equivalence relation is effective (that is, is a kernel pair).

We recall that an equivalence relation on a category $\mathbf{A}$ is a jointly monic pair $\mathbf{r}=\left\langle r_{0}, r_{1}\right\rangle: R \rightarrow X$ such that, for all objects $U$, it induces an equivalence relation on the set $\mathbf{A}(U, X)$. If $\mathbf{A}$ is left exact, this is equivalent to requiring that $\mathbf{r}: R \rightarrow X$ should satisfy the following conditions:

R) there exists a map $r: X \rightarrow R$ such that $r r_{0}=1=r r_{1}$;

S) there exists a map $s: R \rightarrow R$ such that $s r_{0}=r_{1} \& s r_{1}=r_{0}$;

T) if $d_{0}, d_{1}: R * R \rightarrow R$ is the pullback of $r_{0}$ by $r_{1}$ (in particular $d_{1} r_{0}=d_{0} r_{1}$ ), then there exists a map $t: R * R \rightarrow R$ such that $t r_{0}=d_{0} r_{0} \& t r_{1}=d_{1} r_{1}$.

A pseudo equivalence relation is a pair $\mathbf{r}=\left\langle r_{0}, r_{1}\right\rangle: R \rightarrow X$ such that for each $U$ the image of the map $\mathbf{A}(U, R) \rightarrow \mathbf{A}(U, X) \times \mathbf{A}(U, X)$ induced by composition is an equivalence relation on the set $\mathbf{A}(U, X)$. As for the equivalence relations, if $\mathbf{A}$ is a left exact category, the notion of pseudo equivalence relation (p.e.r) is equivalent to the requirements $\mathbf{R}$ ), $\mathbf{S}$ ), T), with the only difference that the maps $r, s, t$ are not necessarily unique anymore. To simplify the understanding let us introduce the following (abuse of) notations for general pseudo relations $\mathbf{r}$ : $R \rightarrow X:$ if $\left\langle x, x^{\prime}\right\rangle: U \rightarrow X$ is a pair of $U$-elements of $X$, we write $x R x^{\prime}$ if there exists $k: U \rightarrow R$ such that $x=k r_{0}$ and $x^{\prime}=k r_{1}$. By the previous definition, $\mathbf{r}$ : $R \rightarrow X$ is a p.e.r. if and only if for each $U$ and for all $U$-elements $x, x^{\prime}, x^{\prime \prime}$, we have: $x R x, x R x^{\prime} \Rightarrow x^{\prime} R x, x R x^{\prime}$ and $x^{\prime} R x^{\prime \prime} \Rightarrow x R x^{\prime \prime}$. Finally, let us remark that a category is exact if and only if it is pseudo exact, that is, condition c) in the definition is replaced by: $c^{\prime}$ ) the regular image of every p.e.r. is effective.

If $\mathbf{A}$ is any category, let us consider the new category $\mathbf{A}_{\mathrm{ex}}$ whose objects are the p.e.r. $\mathbf{r}=\left\langle r_{0}, r_{1}\right\rangle: R \rightarrow X$, which we write as $(X ; R, \mathbf{r})$, and whose arrows ${ }_{\downarrow} f_{1}:(X$; $R, \mathbf{r}) \rightarrow(Y ; S, \mathbf{s})$ are equivalence classes of arrows $f: X \rightarrow Y$ "compatible with the relations on $X$ and $Y$ ", that is, such that for all $U$-elements $x, x^{\prime}: U \rightarrow X$ we have $x f S x^{\prime} f$ or, equivalently, such that there exists $f^{\prime}: R \rightarrow S$ with $r_{0} f=f^{\prime} s_{0}$ and 
$r_{1} f=f^{\prime} s_{1}$; we consider equivalent two such arrows $f, g: X \rightarrow Y$ when, for all $x$ : $U \rightarrow X$ we have $x f S x g$ or, equivalently, when there exists $\Sigma: X \rightarrow S$ such that $\Sigma s_{0}=f$ and $\Sigma s_{1}=g$.

Easily $\mathbf{A}_{\mathrm{ex}}$ is a category, because the relation on maps described is a congruence, and there exists an obvious functor $\Gamma: \mathbf{A} \rightarrow \mathbf{A}_{\mathrm{ex}}$ sending each object to the trivial equivalence relation on it.

In the next section we will show that if $\mathbf{A}$ is a left exact category, then $\mathbf{A}_{\mathrm{ex}}$ is its free exact completion, that is, it is exact and it has the universal property stated in the introduction.

\section{Exactness and universality}

From now on let us suppose $\mathbf{A}$ is a left exact category. In this case can be useful to observe that if $(X ; R, \mathbf{r})$ and $(Y ; S, \mathbf{s})$ are two objects of $\mathbf{A}_{\mathrm{ex}}$, then the product $\mathbf{r} \times \mathbf{s}=\left\langle r_{0} \times s_{0}, r_{1} \times s_{1}\right\rangle: R \times S \rightarrow(X \times Y) \times(X \times Y)$ still is a p.e.r., and if $f: Z \rightarrow X$ is any morphism of $\mathbf{A}$, then the pullback of $\left\langle r_{0}, r_{1}\right\rangle: R \rightarrow X \times X$ along $f \times f$ is also a p.e.r. on $Z$, which we denote $\left(Z ; f^{*} R, \mathbf{r}^{*}\right)$; this can be easily proved by using the previous characterization of p.e.r.'s by $U$-elements. To show that $\mathbf{A}_{\mathrm{ex}}$ is left exact, the naive idea is to handle a new object $(X ; R, \mathbf{r})$ as if it were an old one $X$ of $\mathbf{A}$, but with a new "equality" given by the p.e.r. So, for example, the pullback of two arrows $f_{\lrcorner}:(X ; R, \mathbf{r}) \rightarrow(Z ; T, \mathbf{t})$ and ${ }_{\iota} g_{\lrcorner}:(Y$; $S, \mathbf{s}) \rightarrow(Z ; T, \mathbf{t})$ will have underlying object $X \times{ }_{T} Y$ defined as the pullback of $\left\langle t_{0}, t_{1}\right\rangle: T \rightarrow Z \times Z$ along $f \times g$, and with the p.e.r. given by the pullback of $\left\langle r_{0} \times s_{0}, r_{1} \times s_{1}\right\rangle: R \times S \rightarrow(X \times Y) \times(X \times Y)$ along $t^{*} \times t^{*}$, where $t^{*}: X$ $\times_{T} Y \rightarrow X \times Y$ is the map defined by the first pullback. One can easily check that these definitions do not depend on the choice of $f$ and $g$ in their class and that, together with the definition of the terminal object in $A_{e x}$ (as $\Gamma$ of the terminal in $\mathbf{A}$ ), they yield the left exactness of $\mathbf{A}_{\mathrm{ex}}$ as well that of $\Gamma$.

To show $\mathbf{A}_{\mathrm{ex}}$ has the stated properties, we give the main definitions; details can be found in [3].

a) The regular epi-mono factorization of a morphism ${ }_{\llcorner} f_{\lrcorner}:(X ; R, \mathbf{r}) \rightarrow(Y ; S, \mathbf{s})$ is

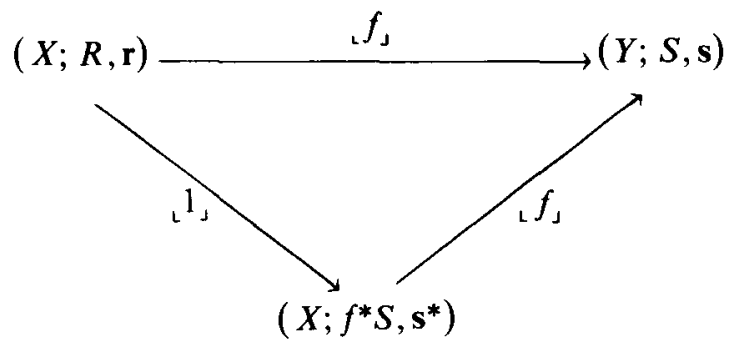


b) By the previous description of the regular epi-mono factorization, it follows that a morphism ${ }_{\llcorner} f_{J}:(X ; R, \mathbf{r}) \rightarrow(Y ; s, \mathbf{s})$ is a regular epi iff ${ }_{\llcorner} f_{\mathrm{J}}:(X ; f S, \mathbf{s}) \rightarrow(Y$; $S, \mathbf{s})$ is an iso, that is, if and only if there exist $s: Y \rightarrow X$ and $\Sigma: Y \rightarrow S$ such that $\Sigma s_{0}=s f$ and $\Sigma s_{1}=1$. So, from the definition of pullbacks in $\mathbf{A}_{\mathrm{ex}}$, condition b) for an exact category follows. It should be observed that a regular epi in $\mathbf{A}_{\text {ex }}$ is always isomorphic in $\mathbf{A}_{\mathrm{ex}}$ to one with the same codomain and whose underlying morphism in $\mathbf{A}$ has a section.

c) A p.e.r. in $\mathbf{A}_{\mathrm{ex}}$ is a pair $\left.\left\langle_{\llcorner} h_{0\lrcorner},{ }_{\downarrow} h_{1}\right\rangle\right):(X ; R, \mathbf{r}) \rightarrow(Y ; S, \mathbf{s})$ satisfying:

R) there exists ${ }_{\llcorner} h_{\lrcorner}:(Y ; S, \mathbf{s}) \rightarrow(X ; R, \mathbf{r})$ such that ${ }_{\llcorner} h h_{0\lrcorner}={ }_{\llcorner} 1, \&_{\llcorner} h h_{1\lrcorner}={ }_{\llcorner} 1_{\lrcorner}$;

S) there exists ${ }_{\llcorner} k_{\lrcorner}:(X ; R, \mathbf{r}) \rightarrow(X ; R, \mathbf{r})$ such that ${ }_{\llcorner} k h_{0\lrcorner}={ }_{\llcorner} h_{1\lrcorner} \&_{\llcorner} k h_{1\lrcorner}={ }_{\llcorner} h_{0\lrcorner}$;

T) if we write ${ }_{\llcorner} d_{0\lrcorner}, d_{1\lrcorner}:\left(X \times_{S} X ; R \times{ }_{S} R, \mathbf{k}\right) \rightarrow(X ; R, \mathbf{r})$ for the pullback in $\mathbf{A}_{\mathrm{ex}}$ of ${ }_{\llcorner} h_{0}$, by ${ }_{L} h_{1\lrcorner}$ (in particular $\left.{ }_{\llcorner} d_{1} h_{0\lrcorner}={ }_{\llcorner} d_{0} h_{1\lrcorner}\right)$, there exists ${ }_{\llcorner} t_{\lrcorner}:\left(X \times{ }_{S} X\right.$; $\left.R \times{ }_{S} R, \mathbf{k}\right) \rightarrow(X ; R, \mathbf{r})$ such that ${ }_{\llcorner} d_{0} h_{0\lrcorner}={ }_{L} t h_{0\lrcorner}$ and ${ }_{\llcorner} d_{1} h_{1\lrcorner}={ }_{L} t h_{1\lrcorner}$. To construct the coequalizer of such a p.e.r., consider the pullbacks:
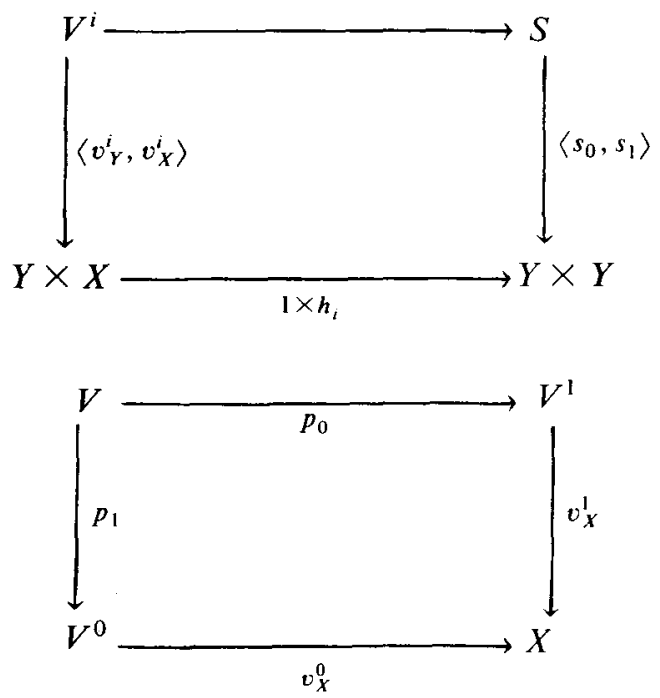

and define $v_{0}, v_{1}: V \rightarrow Y$ as $v_{0}=p_{1} v_{Y}^{0}$ and $v_{1}=p_{0} v_{Y}^{1}$. To show that $(Y ; V, \mathbf{v})$ is an object of $\mathbf{A}_{\mathrm{ex}}$ let us use elements; first, if $y, y^{\prime}: U \rightarrow Y$ are two $U$-elements of $Y$, then:

$y V y^{\prime}$ if and only if there exist $t_{0}: U \rightarrow V^{0}$ and $t_{1}: U \rightarrow V^{1}$ such that $t_{0} v_{X}^{0}=t_{1} v_{X}^{1}$ and $t_{0} v_{Y}^{0}=y, \quad t_{1} v_{Y}^{1}=y^{\prime}$; if and only if there exist $x: U \rightarrow X$ and $k_{0}, k_{1}: U \rightarrow S$ such that $\left\langle y, x h_{0}\right\rangle=k_{0}\left\langle s_{0}, s_{1}\right\rangle$ and $\left\langle y^{\prime}, x h_{1}\right\rangle=k_{1}\left\langle s_{0}, s_{1}\right\rangle$; if and only if there exists $x: U \rightarrow X$ such that $y S x h_{0}$ and $y^{\prime} S x h_{1}$. 
Now, to show conditions $\mathbf{R}), \mathbf{S})$ and $\mathbf{T})$ for $(Y ; V, \mathbf{v})$ is straightforward. For example, let us show $\mathbf{T}$ ). Let $y V y^{\prime}$ and $y^{\prime} V y^{\prime \prime}$; then there exists $x: U \rightarrow X$ such that $y S x h_{0}$ and $y^{\prime} S x h_{1}$ and there exists $x^{\prime}: U \rightarrow X$ such that $y^{\prime} S x^{\prime} h_{0}$ and $y^{\prime \prime} S x^{\prime} h_{1}$. Because of condition $\mathbf{T})$ for $(Y ; S, \mathbf{s})$, we have $x^{\prime} h_{0} S x h_{1}$; therefore there exists $\bar{x}$ : $U \rightarrow: U \rightarrow X \times{ }_{S} X$ such that $x=\bar{x} d_{1}$ and $x^{\prime}=\bar{x} d_{0}$; because of condition $\mathbf{T}$ ) for ${ }_{\llcorner} h_{0\lrcorner},{ }_{\llcorner} h_{1\lrcorner}, x^{\prime \prime}=\bar{x} t$ is such that $x^{\prime \prime} h_{0} S x h_{0}$ and $x^{\prime \prime} h_{1} S x^{\prime} h_{1} ;$ so, $y S x^{\prime \prime} h_{0}$ and $y^{\prime \prime} S x^{\prime \prime} h_{1}$, which means $y V y^{\prime \prime}$. In the same way, one can prove that ${ }_{\llcorner} 1_{\perp}:(Y$; $S, \mathbf{s}) \rightarrow(Y ; V, \mathbf{v})$ is an arrow of $\mathbf{A}_{\text {ex }}$ and that $\operatorname{ker}\left({ }_{L}{ }_{1}:(Y ; S, \mathbf{s}) \rightarrow(Y ; V, \mathbf{v})\right)$ is isomorphic to $\operatorname{Im}\left(\left\langle_{\llcorner} h_{0\lrcorner},{ }_{\llcorner} h_{1\lrcorner}\right\rangle:(X ; R, \mathbf{r}) \rightarrow(Y ; S, \mathbf{s}) \times(Y ; S, \mathbf{s})\right)$, that is, condition $\mathrm{c}^{\prime}$ ).

To show the universal property of $\Gamma: \mathbf{A} \rightarrow \mathbf{A}_{\mathrm{ex}}$ with respect to left exact functors $F$ from $\mathbf{A}$ to any exact category $\mathbb{E}$, first observe that any object $(X ; R, \mathbf{r})$ of $\mathbf{A}_{\text {ex }}$ appears as the coequalizer of $\Gamma r_{0}, \Gamma r_{1}: \Gamma R \rightarrow \Gamma X$ and any morphism $f_{\lrcorner}$: $(X ; R, \mathbf{r}) \rightarrow(Y ; S, \mathbf{s})$ appears in $\mathbf{A}_{\mathrm{ex}}$ as the unique extension of $f_{\lrcorner}$to the quotients of $\Gamma r_{0}, \Gamma r_{1}$ and $\Gamma s_{0}, \Gamma s_{1}$; the condition on the extension $\hat{F}: \mathbf{A}_{\mathrm{ex}} \rightarrow \mathbb{E}$ to be exact forces us to define it on objects as

$$
\hat{F}(X ; R, \mathbf{r})=\operatorname{Coeq}\left(F r_{0}, F r_{1}: F R \rightarrow F X\right)
$$

and similarly on morphisms.

Because $F$ is left exact and because $\mathbb{E}$ is exact, $\hat{F}$ is well defined and by the previous observation it is unique up to isomorphisms, provided it is exact. It is easy to see that $\hat{F}$ preserves regular images. A bit more laborious is the verification of left exactness of $\hat{F}$. The following lemma is basically what we need to prove it.

\section{LEMMA. $\hat{F}$ preserves mono.}

Proof. The proof reduces to observing that $f_{1}:(X ; R, \mathbf{r}) \rightarrow(Y ; S, \mathbf{s})$ is mono if and only if ${ }_{\mathrm{L}}{ }_{\lrcorner}:(X ; R, \mathbf{r}) \rightarrow\left(X ; f^{*} R, \mathbf{r}^{*}\right)$ is an iso in $\mathbf{A}_{\mathrm{ex}}$, and to prove that in an exact category if $p: U \rightarrow U^{\prime}$ and $q: V \rightarrow V^{\prime}$ are regular epis and $f: U \rightarrow V$ is a map such that the pullback of $\operatorname{ker}(q) \rightarrow V \times V$ along $f \times f$ is $\operatorname{ker}(p) \rightarrow U \times U$, then the map $f^{\prime}: U^{\prime} \rightarrow V^{\prime}$ induced by $f$ is mono.

The following lemma, whose proof should now be easy, is basically what we need to prove that the endofunctor on Eex defined by the construction $A_{\mathrm{ex}}$ is in fact a Kock-Zöberlein doctrine (see [4]): 
Lemma. Let $\mathbf{A}$ be a left exact category and let $\Gamma: \mathbf{A} \rightarrow \mathbf{A}_{\mathrm{ex}}$ be the left exact embedding previously defined. Then, $\mathbf{A}$ is an exact category if and only if $\Gamma$ has a left exact left adjoint.

\section{The free abelian category}

From the construction of the last section, it is clear that if $\mathbf{A}$ is a left exact and additive category, then the exact completion $\mathbf{A}_{\mathrm{ex}}$ still is additive; so, by Tierney's theorem quoted in the introduction, $\mathbf{A}_{\mathrm{cx}}$ is abelian and, in fact, $\mathbf{A}_{\mathrm{ex}}$ is the free abelian category on the additive and left exact (= with kernels) category $\mathbf{A}$. The only thing to check is that the equivalence relation between the morphisms is a congruence with respect to the additive structure on $\mathbf{A}$, so that the additive structure on $\mathbf{A}_{\mathrm{ex}}$ can be defined naturally as $f_{\llcorner} f_{\lrcorner} g_{\lrcorner}={ }_{\llcorner} f+g_{\lrcorner}$and everything else works. Now, if we want to construct the free abelian category on an additive one, all we need to do is to freely add kernels to an additive category and then to apply the previous construction of the exact completion.

The free additive category with kernels $\boldsymbol{A}_{\text {ker }}$ on an additive category $\mathbf{A}$ can be constructed as follows: objects are arrows $\alpha: X \rightarrow X^{\prime}$ of $\mathbf{A}$, which we denote by $\left(X, X^{\prime}, \alpha\right)$ : arrows ${ }_{\llcorner} f_{\jmath}:\left(X, X^{\prime}, \alpha\right) \rightarrow\left(Y, Y^{\prime}, \beta\right)$ are equivalence classes of arrows $f$ : $X \rightarrow Y$ of A such that there exists $f^{\prime}: X^{\prime} \rightarrow Y^{\prime}$ with $f \beta=\alpha f^{\prime}$; we consider equivalent two such arrows $f, g: X \rightarrow Y$ when "they have the same restriction to the kernels", that is when there exists $\Sigma: X^{\prime} \rightarrow Y$ such that $\alpha \Sigma=f-g$. the relation between morphisms being homotopy, $\boldsymbol{A}_{\text {ker }}$ is an additive category. One can easily show that the kernel of a morphism ${ }_{\llcorner} f_{\lrcorner}:\left(X, X^{\prime}, \alpha\right) \rightarrow\left(Y, Y^{\prime}, \beta\right)$ can be defined as 1: $\left(X, Y \times X^{\prime},\langle f, \alpha\rangle\right) \rightarrow\left(X, X^{\prime}, \alpha\right)$. Thus $\mathbf{A}_{\mathrm{ker}}$ is an additive category with kernels.

There exists an obvious additive functor $\Gamma: \mathbf{A} \rightarrow \mathbf{A}_{\mathrm{ker}}$ sending each object $X$ of $A$ in $0: X \rightarrow X$, which gives rise to an equivalence of categories between the category of additive functors from $\mathbf{A}$ to $\mathbf{B}$ ( $\mathbf{B}$ being an additive category with kernels) and the category of additive kernel preserving functors from $\mathbf{A}_{\text {ker }}$ to $\mathbf{B}$. For the proof it suffices to observe that each object $\left(X, X^{\prime}, \alpha\right)$ of $\mathbf{A}_{\mathrm{ker}}$ is isomorphic to the kernel $\left(X, X^{\prime},\langle\alpha, 0\rangle\right)$ of $\Gamma \alpha: \Gamma X \rightarrow \Gamma X^{\prime}$, and that each arrow $f_{\lrcorner}:\left(X, X^{\prime}, \boldsymbol{\alpha}\right) \rightarrow\left(Y, Y^{\prime}, \boldsymbol{\beta}\right)$ appears in $\mathbf{A}_{\text {ker }}$ as the unique restriction to $\operatorname{ker}(\Gamma \boldsymbol{\alpha})$ and $\operatorname{ker}(\Gamma \beta)$. This observation forces us to define the extension $\hat{F}: \mathbf{A}_{\mathrm{ker}} \rightarrow \mathbf{B}$ of an additive functor $F$ from $\mathbf{A}$ to an additive category with kernels $\mathbf{B}$, as $\hat{F}\left(X, X^{\prime}, \alpha\right)$ $=\operatorname{ker}(F \boldsymbol{\alpha})$ and similarly on morphisms. With a little effort one can check that $\hat{F}$ is additive and preserves kernels. So, by starting with an additive category $\mathbf{A}$, we can describe the free abelian on it as $\mathbf{A}_{\mathrm{ab}}=\left(\mathbf{A}_{\mathrm{ker}}\right)_{\mathrm{ex}}$, which therefore must be equivalent to that constructed in [1]. 


\section{References}

[1] M. Adelman, 'Abelian categories over additive ones,' J. Pure Appl. Algebra 3 (1973), 103-117.

[2] M. Barr, Exact categories and categories of sheaves, pp. 1-120 (Springer Lecture Notes in Math. $236(1971))$.

[3] R. Celia Magno (Thesis, University of Calabria, Cosenza, Italy (1981)).

[4] R. H. Street, 'Fibrations in bicategories,' Cahiers Topologie Géom. Différentielle 21 (1980), $111-160$.

\section{Istituto Matematico}

\section{via C. Saldini 50}

20133 Milano

Italia 